\title{
Avaliação do crescimento e alometria de Caiman latirostris (Crocodylia, Alligatoridae) em condições seminaturais de manejo.
}

\section{Evaluation of the growth and alometry of Caiman latirostris (Daudin, 1802) in seminatural conditions.}

\author{
Mauro Sergio Cruz Souza Lima ${ }^{1}$ \\ Jonas Pederassi \\ Carlos Alberto Santos Souza ${ }^{3}$
}

Palavras-chave

Crocodylia

Jacaré-de-papoamarelo

Alometria

Reprodução

\section{Resumo}

Foi avaliada a taxa de eclodibilidade e crescimento de uma ninhada de Caiman latirostris por 80 dias. Os ovos foram coletados e transportados em bandejas com serapilheira do local, observando-se a disposição no ninho para replicação. Os animais foram levados ao laboratório e dispostos individualmente em caixas de isopor com substrato inclinado, proporcionando $2 / 3$ de área seca e $1 / 3$ de área úmida. $\mathrm{O}$ substrato foi composto de areia, serapilheira e água. Avaliou-se a morfometria dos animais no nascimento, aos 40 e 80 dias da eclosão. As variáveis corpóreas foram analisadas pela alometria multivariada através da Análise de Componentes Principais e suas correlações foram testadas pela correlação de Spearman além de analisadas pelo Box Plot de modo a avaliar a correlação das variáveis entre si. Obteve-se um sucesso de $91 \%$ na taxa de eclodibilidade, tendo os filhotes uma taxa média de crescimento de 0,66 $\mathrm{cm}(\bar{x}=0,66 \mathrm{~cm} ; \mathrm{DP}=0,01)$. Pela análise do Box Plot a cabeça é, dentre as variáveis observadas, a região com menor variação além de ser a porção do corpo com retardo de crescimento, de acordo com a Análise dos Componentes Principais e quando comparados os três segmentos corpóreos com o comprimento total a cabeça apresentou alometria negativa enquanto o tronco e a cauda têm alometria positiva, não sendo observado crescimento isométrico algum. Ao final de 90 dias, todos os filhotes que eclodiram foram levados ao local de origem para soltura, sob os cuidados da Polícia Florestal do Estado de São Paulo, Brasil.

\section{Abstract}

The eclodibility, development and allometry of a brood of Caiman latirostris were evaluated for 80 days. The eggs were collected and transported in trays with leaf litter of the place, being observed the disposition in the nest for further replication. The individuals were taken to the laboratory and disposed individually in Styrofoam boxes with sloping substratum providing 2/3 of dry area and 1/3 of humid area, the substratum was composed of sand, leaf litter and water. The youths were fed with earthworms Eisenia foetida whose fossorial habit stimulates the natural behavior of the alligators of this species, facilitating their reintroduction to the nature. The biometry of the animals were taken in the birth, 40 and 80 days after hatchling, the variables were analyzed by Multivariated Allometry through the Analysis of Main Components and their correlations were tested by the correlation of Spearman. In addition they were analyzed by the Box Plot in order to evaluate the correlation of the variables among themselves. A success of $91 \%$ in the eclodibility was obtained, having the nestlings an average of growth of $0.66 \mathrm{~cm}$ and $S D=0.01$. For Box Plot analysis the head is, among the observed variables, the body part with smaller variation, besides, it is the portion of the body with

\section{Key words}

Crocodylia

Broad-snouted caiman

Biometry

Reproduction
Artigo

Original

Original

Paper

Recebido em 05/2012

Aprovado em $08 / 2012$

\footnotetext{
${ }^{1}$ UFPI - Universidade Federal do Piauí - Campus Amilcar Ferreira Sobral 
growth retard, in agreement with the Analysis of the Main Components and when compared the three corporeal segments with the total length, the head presented negative allometry while the trunk and the tail had positive allometry, not being observed any isometric growth. At the end of 90 days, all the alligators that have emerged were taken to the original place for looseness, under the cares of the Forest Police of the State of São Paulo, Brazil.

\section{Introdução}

O jacaré Caiman latirostris Daudin, 1802 apresenta distribuição geográfica ampla, que vai da região nordeste do Brasil ao extremo sudeste da Bolívia, incluindo o norte da Argentina, Uruguai, Paraguai e as bacias hidrográficas dos rios Paraná e São Francisco, além das pequenas bacias costeiras do leste do Brasil(GROOMBRIDGE, 1982; BRAZAITIS et al., 1990). A destruição de seus habitats e, em segundo plano, a caça têm sido as principais causas de seu declínio populacional em praticamente toda sua área de distribuição geográfica. Atualmente, suas populações selvagens remanescentes encontram-se fragmentadas, porém, já são classificadas com risco de extinção pouco preocupante (IUCN, 2010).

As taxas de crescimento são extremamente variáveis dentro e entre as diferentes espécies de crocodilianos devido a fatores como variabilidade genética, clima, suprimento alimentar, densidade populacional e carga parasitária (DE VOS, 1982), época de nascimento (MAGNUSSON; TAYLOR, 1981; MESSEL; VORLICEK, 1984) e época do ano (CHABRECK; JOANEN, 1979) e as relações alométricas podem ser úteis na avaliação do tamanho corporal a partir de partes isoladas do corpo (SCHMIDT-NIELSEN, 1984), como nos monitoramentos de populações de crocodilianos, onde usualmente indivíduos são contados quando apenas suas cabeças estão visíveis, logo, a relação entre o tamanho da cabeça e o comprimento total do corpo é utilizada para estabelecer a distribuição entre classes por tamanho (VERDADE, 2000). No presente estudo observamos o crescimento e a alometria corpórea de uma ninhada de $C$. latirostris, com o objetivo de avaliar a eficácia do manejo empregado, necessário à sobrevivência dos filhotes, em que a alimentação provida com minhocas Eisenia foetida buscou manter o instinto natural de forrageio da espécie de modo a aumentar a viabilidade dos filhotes após a soltura.

\section{Material e Métodos}

\subsection{Licença de Coleta}

Caiman latirostris é espécie silvestre que exige a licença obrigatória do órgão federal (IBAMA, 2009) para seu estudo e manejo. Neste caso, o batalhão florestal de São Paulo prendeu um grupo de caçadores ilegais que mataram a fêmea que nidificava, sendo necessário cuidado para com o ninho, sob pena dos ovos serem predados ou coletados pela população local. O ninho, com 35 ovos, ocupava um fragmento de floresta Atlântica, no município de Bananal, interior de São Paulo, Brasil. Findo 90 dias de cativeiro, os animais foram levados para o local da coleta sob acompanhamento do batalhão florestal onde os filhotes foram soltos.

\subsection{Conservação das características do ninho natural}

O ninho era formado por "murundum de folhas" de $75 \mathrm{~cm}$, com camadas de serapilheira cobrindo os ovos. Neste caso, cada camada de ovos foi descoberta e colocada em bandejas plásticas ( 7 × $20 \times 35 \mathrm{~cm})$ com serapilheira do local, observando-se a manutenção da disposição de oviposição, sendo então colocados em caixa de isopor e transportadas para fazenda experimental da Universidade Federal Fluminense, distante $30 \mathrm{~km}$ do local de nidificação, onde foi construído um ninho com serapilheira obedecendo às dimensões do ninho natural $(0,75 \mathrm{~m}$ de altura por 1,20 m de diâmetro em sua base). Ao redor deste foi construído uma estrutura de bambu que gerasse sombra e proteção contra pisoteio por qualquer animal de grande porte.

$O$ período de incubação não foi determinado por não ter sido possível saber o dia da postura, porém, os ovos estiveram incubados por 25 dias desde a data do transporte até o momento da vocalização dos filhotes, que, após eclosão, foram transportados para o laborató- 
rio e mantidos individualmente em caixa de isopor $(20 \times 25 \times 30 \mathrm{~cm})$ com substrato inclinado, proporcionando $2 / 3$ de área seca para $1 / 3$ de área úmida, sendo o substrato composto por areia, serapilheira e água. A limpeza dos recintos foi diária com reposição de areia e água.

Os filhotes foram alimentados diariamente com minhocas vivas (Eisenia foetida) oriundas de minhocários comerciais. Cada minhoca pesava em média $0,5 \mathrm{~g}$ o que correspondia a aproximadamente de 300 a 400 gramas semanais de ingestão de alimento por animal.

Os jacarés foram medidos e pesados no nascimento, aos 40 dias, 60 dias e 80 dias. A morfometria correspondeu ao comprimento da cauda (CAU), comprimento do tronco (CORP) e comprimento da cabeça (CAB). As medidas foram realizadas com paquímetro de acuracidade de $0,03 \mathrm{~mm}$ e o peso foi mensurado com balança digital com precisão de um grama.

\subsection{Análise estatística}

As variáveis corpóreas foram submetidas à alometria multivariada em que a relação entre CAU, CORP e CAB foram avaliados pela Análise dos Componentes Principais. A hipótese de isometria do vetor crescimento das variáveis corpóreas foi verificada com o teste: Isometria $=\sqrt{1 / p}$. Sendo $p=$ número de variáveis, com $p-1$ graus de liberdade (GL). O vetor maior que $\sqrt{1 / p}$ indica alometria positiva e menor, negativa (MANDARINDE-LACERDA, 1995). A associação entre as variáveis foi estabelecida pelo Coeficiente de Correlação de Spearman $\left(\mathrm{r}^{2}\right)$. As variáveis foram submetidas também à análise Box Plot para avaliação do grau de correlação de crescimento entre elas e o crescimento total.

\section{Resultados e Discussão}

A incubação de ovos em condições artificiais exige medidas urgentes de conhecimento para evitar perdas desnecessárias de embriões ou mesmo ninhadas inteiras (VERDADE; MOLINA, 1993; VERDADE et al., 1993). Neste caso, a ninhada total estudada correspondeu a 35 ovos. O sucesso de eclodibilidade alcançou 91\% (32 ovos), sendo mantidos os ovos em condições experimentais semelhantes às condições naturais. Os $9 \%$ de insucesso, que corresponderam a três ovos em estado de putrefação, pode ter sido acarretado pela disposição destes no ninho experimental que diferiam do ninho original na constituição da serapilheira, isto é, no ninho experimental utilizou-se palha de bambu para gerar a sombra, enquanto no ninho natural, a sombra era gerada por árvores de grande porte. Outro fator a ser considerado é o número de perdas representar uma taxa natural por má formação ou outro processo natural que inviabilize a sobrevivência do embrião.

Não foi possível estabelecer o sexo dos filhotes, no entanto, a manutenção do ninho experimental respeitou as condições físicas naturais encontradas com camadas de ovos e serapilheira dispostas $20 \mathrm{~cm}$ umas das outras, com cerca de sete a nove ovos, separados por serapilheira e com porção terminal do ninho a $23 \mathrm{~cm}$ da superfície. Esse pode ter sido um dos fatores preponderantes para os $91 \%$ de sucesso de eclodibilidade.

Em cativeiro, os ovos podem ser incubados em caixas de isopor ou alvenaria e dispostos em vermiculita com a temperatura local controlada em ambientes fechados que não sofram a influência do ambiente externo (VERDADE et al., 1992). O material vegetal do ninho tem, no entanto, a vantagem de apresentar um pH mais ácido devido à liberação de ácidos metabólicos bacterianos, que provocam uma progressiva dissolução dos cristais de carbonato de cálcio da casca do ovo, diminuindo sua resistência por ocasião da eclosão (FERGUSON, 1981).

No fim do período de incubação os embriões emitem vocalizações características, ainda no interior dos ovos, que aparentemente têm a função de estimular toda a ninhada a eclodir em conjunto e também estimular à mãe a abrir o ninho (LEE, 1968). Neste estudo a manutenção dos ovos em serapilheira contribuiu para diminuição da resistência dos ovos no momento da eclosão, momento em que ocorreu o início da vocalização e os filhotes receberam o auxílio manual na retirada inicial da casca do ovo.

Considerando os caracteres comportamentais do jacaré-de-papo-amarelo, optou-se por constituir um ambiente individual em caixa de isopor que impedisse comportamento antagônico intraespecífico e, ao mesmo tempo configurasse dois ambientes, um seco e um úmido. $\mathrm{O}$ 
alimento ofertado foi minhoca (Eisenia foetida) uma vez que estes animais são crípticos e exigiam dos filhotes o instinto de cavar em busca do alimento. Dessa forma, foi mantido o instinto natural da espécie, uma vez que o objetivo principal era retornar o animal para as condições naturais, pois segundo Diefenbach (1979) o comportamento característico dessa espécie de escavar o leito dos corpos d'água em que habita pode estar associado à captura de invertebrados, constituintes importantes de sua dieta. A correlação para os 32 indivíduos e cinco intervalos de tempo, desde o nascimento até os 80 dias de vida e ganho de peso, correspondeu a $98 \%$ com $\mathrm{r}^{2}$ variando entre 0,97 e $0,99(\mathrm{p}<0,001)$. Sendo que o peso variou entre 35 e 49 gramas ( 1 dia: $\bar{x}=35,04 \pm 2,32 \mathrm{~g} ; 20$ dias: $\bar{x}=38,56$ $\pm 2,35$ g; 40 dias: $\bar{x}=44,12 \pm 3,10$ g; 60 dias: $\bar{x}$ $=47,88 \pm 3,83 \mathrm{~g} ; 80$ dias: $\bar{x}=49,61 \pm 2,91 \mathrm{~g}$ ),

(Figura 1).

Ganho de Peso (Box Plot)

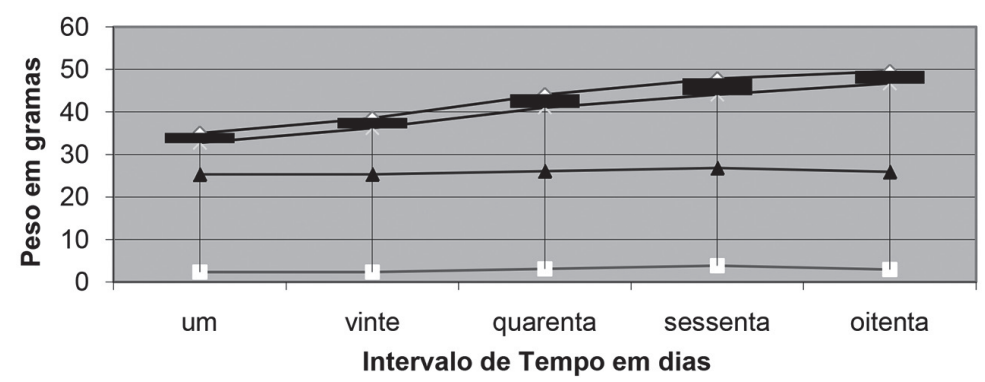

Figura 1- Correlação entre o ganho de peso e maturidade corpórea (Box Plot)

Em cativeiro, os fatores que podem interferir na taxa de crescimento de uma mesma espécie são: dieta, variabilidade genética, tamanho e origem dos animais em questão (DE VOS, 1982; GARNETT; MURRAY, 1986). Os crocodilianos quando bem alimentados crescem rapidamente e, sob condições ideais, podem alcançar um metro ou mais em um ano e 1,5 m em dois anos (CARDEILHAC; JOANEN, 1989). Montague (1982) relata uma taxa de crescimento de 15,7 a $17,0 \mathrm{~cm}$ por ano para, respectivamente, fêmeas e machos de Crocodylus novaeguineae. Bustard e Singh (1980) relatam um crescimento médio de $49 \mathrm{~cm}$ por ano para Gavialis gangeticus nos primeiros quatro e meio anos de vida em ambiente semi- natural, enquanto para $C$. latirostris, em um período de treze meses, Pinheiro e Lavorenti (2001) reportam um crescimento de $75,5 \mathrm{~cm}$ e Larriera (1990) um crescimento de 41,3 cm, ambos em condições de manejo em cativeiro.

A taxa de crescimento diário dos 32 filhotes variou entre 0,58 e $0,70 \mathrm{~cm}(\bar{x}=0,66 \pm$ $0,01)$ por um período de 80 dias o que foi uma taxa muito maior que a prevista por Montague (1982). Ao avaliarmos a curva de crescimento corporal de cada um dos 32 filhotes, durante os 80 dias de experimento, é possível aferir que a taxa de crescimento é maior nos primeiros 20 dias $(0,65$ e $0,71 \mathrm{~cm} ; \bar{x}=0,69 \pm 0,01)$, ente 40 e 80 dias o crescimento é menor $(0,70$ e $0,71 \mathrm{~cm} ; \bar{x}=0,70 \pm 0,01),($ Figura 2$)$

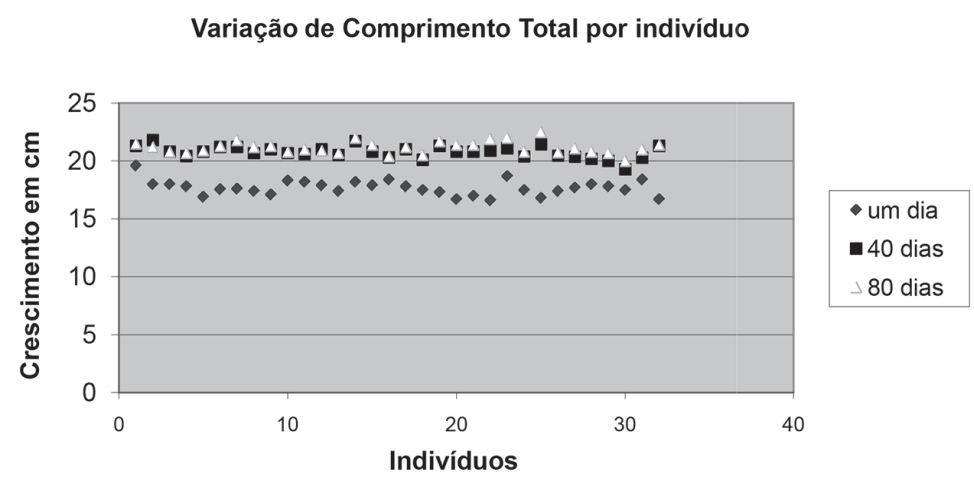

Figura 2 - Crescimento Total dos Filhotes durante o período do nascimento, 40 dias e 80 dias de vida 
Segundo Ferguson (1983), Caiman crocodilus apresenta uma correlação positiva de 0,53 entre a maturidade e o comprimento total, neste estudo a variação encontrada entre o nascimento, 40 e 80 dias foi respectivamente: $\mathrm{r}^{2}=0,13 ; \mathrm{r}^{2}=0,18$ e $\mathrm{r}^{2}=0,76$. De acordo com estes dados C. latirostris apresenta maior correlação de comprimento total que $C$. crocodilus conforme se desenvolve, ainda que, nas fases iniciais, nascimento e 40 dias, a correlação seja menor que de $C$. crocodilus em sua fase adulta. Como o trabalho de Ferguson
(1985) não estabelece correlação para as fases iniciais, provavelmente essa relação de maior correlação entre maturidade e comprimento total prevalece para $C$. latirostris. O conjunto de dados submetidos à análise Box Plot depreende que a cabeça é a região corpórea com menor variação, a cauda e tronco são as regiões com maior variação de crescimento durante os 80 dias de estudo. É possível também identificar que a região corpórea cauda e corpo alteram sua morfologia com forte correlação entre elas $\left(r^{2}=0,74\right.$; Figura 3).

\section{Box Plot - Morfometria}

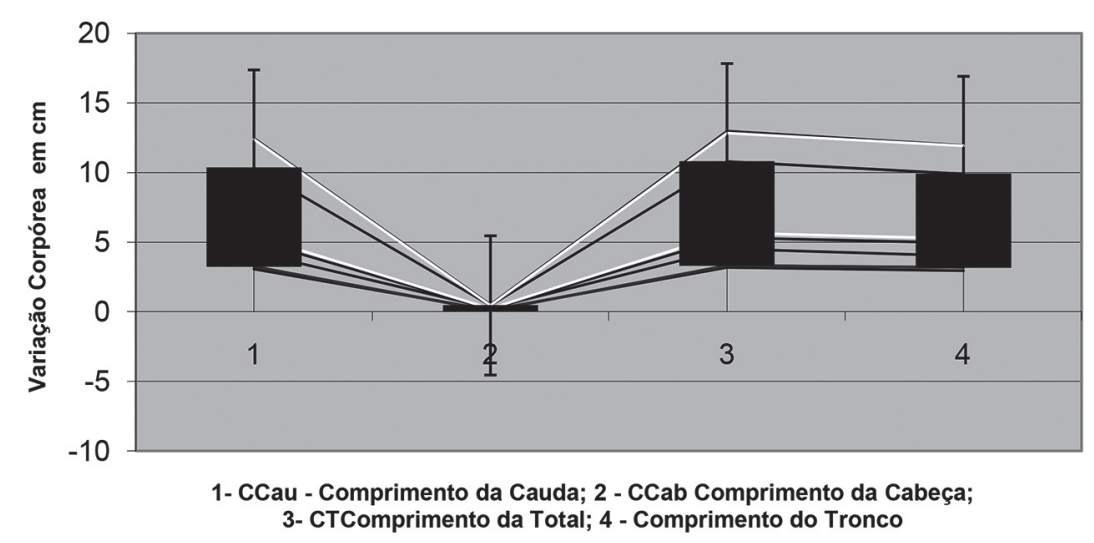

Figura 3 - Análise Morfométrica do crescimento da cabeça, cauda e corpo submetido ao box plot.

Quando a taxa de crescimento dos segmentos corpóreos de $C$. latirostris foi submetida à análise de seus componentes principais, verificou-se que o comprimento da cauda, comprimento do tronco e comprimento da cabeça apresentam pequena variância entre si demonstrando que a cabeça é a região corpórea com retardo de crescimento em relação aos 80 dias de estudo (Figura 4).

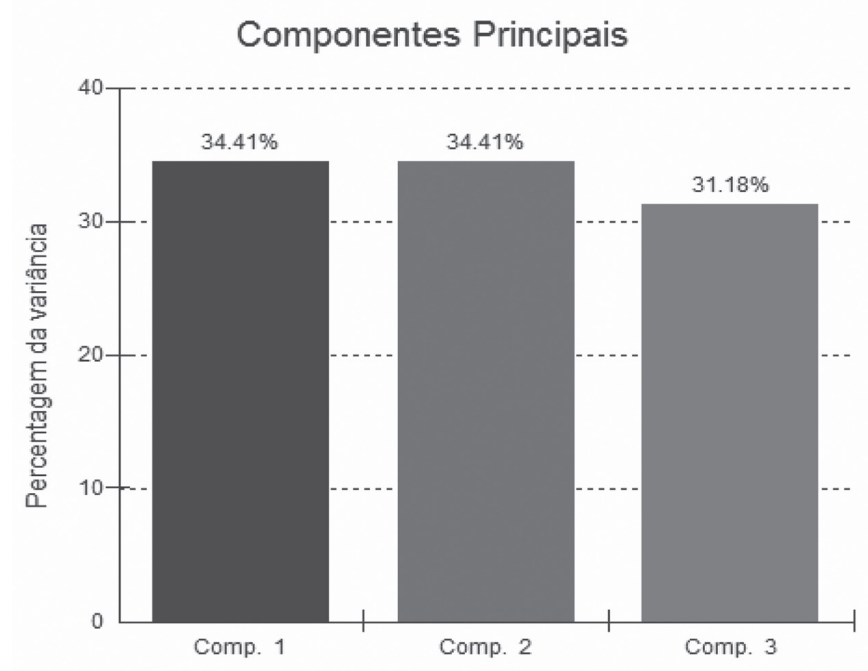

Figura 4 - Análise dos Componentes Principais (ACP) entre o comprimento da cauda (Comp. 1 C.Caud), comprimento do tronco (Comp. 2 - C.corp) e comprimento da cabeça (Comp. 3 - C.cab).

Quando avaliados os três segmentos corporais com o crescimento corpóreo total, encontraram-se resultados que indicam que tronco e cauda crescem com alometria positiva (abaixo de 0,3835). Enquanto, a cabeça cresce com alometria negativa $(-0,6616)$. Nenhuma medida apre- 
sentou crescimento isométrico. Segundo Pough et al. (2008), de forma geral, poucos componentes corpóreos apresentam isometria. $\mathrm{O}$ segmento corporal que apresentou a maior taxa de cresci- mento no período de estudo foi o comprimento da cauda (autovalor $=1,4519$ ), praticamente $30 \%$ maior que a menor taxa de crescimento que foi a do comprimento da cabeça (Tabela 1; Figura 5).

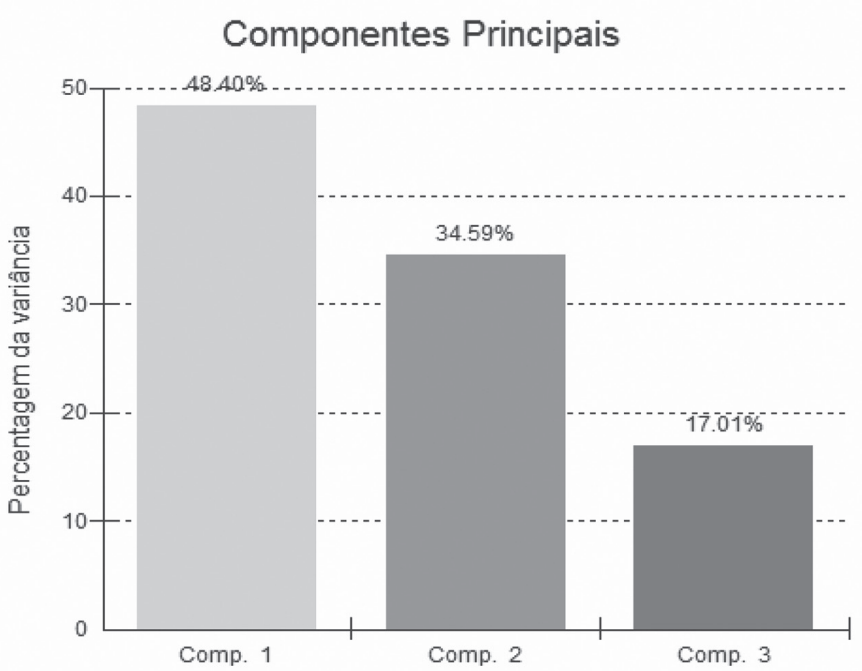

Figura 5 - Análise dos Componentes Principais (ACP). Comp. 1 - Comprimento da Cauda; Comp. 2 - Comprimento do Tronco; Comp. 3 - Comprimento da Cabeça em relação ao crescimento corpóreo total durante os 80 dias de estudo.

\begin{tabular}{|c|c|c|c|}
\hline Coefs. Autovetores & Coef. $\boldsymbol{X 1}$ & Coef. $\boldsymbol{X 2}$ & Coef. X3 \\
\hline Componente 1 & 0.2138 & 0.7187 & -0.6616 \\
\hline Componente 2 & 0.9202 & 0.0792 & 0.3835. \\
\hline Componente 3 & 0.3280 & 0.6908 & 064444 \\
\hline
\end{tabular}

Tabela 1: Análise dos componentes principais.

A metodologia empregada no manejo dos ovos e filhotes mostrou-se adequada tanto em relação ao nível de eclodibilidade quanto ao desenvolvimento dos animais, enquanto a avaliação alométrica evidenciou que não há desenvolvimento isométrico dos segmentos e sim um crescimento mais lento da cabeça, com alometria negativa em relação aos demais segmentos, sendo a cauda a porção com crescimento mais pronunciado.

\section{Agradecimentos}

Os autores agradecem ao Batalhão de Polícia Florestal do Estado de São Paulo, Brasil, pela confiança na entrega da guarda e manutenção da ninhada e acompanhamento na coleta dos ovos e posterior soltura dos filhotes. 


\section{Referências}

1. BRAZAITIS, P.; YAMASHITA, C.; REBELO, C. 1990. A summary report of the CITES central South American caiman study. Phase I: Brazil. p. 100-115. In: Crocodiles. Proc. 9th Work. Meet. Croc. Spec. Group. Vol. 1. IUCN - The World Conservation Union. Glan, Switzerland. p. 1-399.

2. BUSTARD, H.R.; SINGH, L.A.K. 1980. Growth in the gharial. British J. Herpet. 6(3) 107.

3. CARDEILHAC, P.T.; JOANEN, T. 1989. Reproduction, development and growth of the American alligator. p.14. In: Crocodilian Congress, production \& marketing: strategies for the 1990's. Tampa, Florida. :1-147.

4. CHABRECK, R.H.; JOANEN, T. 1979. Growth rates of American alligator in Louisiana. Herpetologica 35(1) :51-57.

5. DE VOS, A. 1982. A manual on crocodile conservation and management in India. FAO Project IND/82/003. Dehra Dun, India.

6. DIEFENBACH, C.O. 1979. Ampullarid gastropod - staple food of Caiman latirostris ?. Copeia (1) 1979:162- 163.

7. FERGUSON, M.W.J. 1985. Reproductive biology and embryology of the crocodilians. p. 329-491. In: GANS, C.; BILLETT, F.; MADERSON, P.F.A [Eds.]. Biology of the Reptilia. Vol. 14. Development A. John Wiley. New York. p. 1-763.

8. FERGUSON, M.W.J.; JOANEN, T. 1983. Temperatura-dependent sex determination in Alligator mississipiensis. $\mathbf{J}$. Zool. Lond. 200:143-177.

9. FERGUSON, M.W.J. 1981. Extrinsic microbial degradation of the alligator eggshell. Science, N.Y. 214(4525):1135-1137.
10. GARNETT, S.T.; MURRAY, R.M. 1986. Parameters affecting the growth of the estuarine crocodile, Crocodylus porosus, in captivity. Australian J. Zool. 34(2) :211-223.

11. GROOMBRIDGE, B. 1982. IUCN Amphibia - Reptilia Red Data Book Part 1: Testudines, Crocodylia, Rhynchocephalia. IUCN Conservation Monitoring Centre. Cambridge.

12. IBAMA - INSTITUTOBRASILEIRODO MEIO AMBIENTE E DOS RECURSOS NATURAIS RENOVÁVEIS. 2009. Portaria $\mathrm{n}^{\mathrm{0}} 1.522$, de 19 de dezembro de 1.989. Disponível em: <www.ibama.gov. br/fauna/legislacao/port_1522_89.pdf >. Acesso em: 24 de junho de 2009.

13. IUCN. 2010. IUCN Red List of Threatened Species. Version 2010.3. Disponível em: <www.iucnredlist.org>. Acessado em 11 Octorber 2010.

14. LARRIERA, A. 1990. Observaciones sobre el crecimiento de Caiman latirostris (Daudin, 1802) nacidos en cautiverio. Peso y longitud a los 30 meses de edad (Crocodylia: Alligatoridae). Amphibia y Reptilia (conservación), mayo, 1(6): 118-119.

15. LEE, D.S. 1968. Possible communication between eggs of the American alligator. Herpetologica 24:88.

16. MAGNUSSON, W.E.; TAYLOR, J.A. 1981 Growth of juvenile Crocodylus porosus as effected by season of hatching. Journal Herpet. 15(2) :242- 245.

17. MANDARIN-DE-LACERDA, C. A. 1995. Métodos Quantitativos em Morfologia. EDUERJ.

18. MESSEL, H.; VORLICEK, G.C. 1984 A review of the growth of Crocodylus porosus in the Northern Australia. p.171215. In:Proc. 6th work. meet. croc. spec. IUCN. Publs (NS) :1- 219. 
19. MONTAGUE, J.J. 1982. Morphology, injury and growth analysis of Crocodylus novaeguineae from the Fly River drainage, Papua New Guinea. Dissertation Abstr. int. (B) 43(5)1315-1316.

20. PINHEIRO, M. S.; LAVORENTI, A. 2001. Growth of broad-nosed caiman, Caiman latirostris (Daudin, 1802) hatchlings, fed with diets of animal origin. Braz. J. Biol., 61(3): 421-429.

21. POUGH, H. F.; JANIS, C. M.; HEISER, J. B. 2008. A vida dos vertebrados. ed. 4 Atheneu. São Paulo. 739 p.

22. SCHMIDT-NIELSEN, K. 1984. Scaling: Why is Animal Size so Important? Cambridge University Press, Cambridge.

23. VERDADE, L. M. 1992. Manejo reprodutivo do jacaré-de-papo-amarelo, Caiman latirostris (Daudin, 1802) em cativeiro. Dissertação apresentada à Escola Superior de Agricultura "Luiz de Queiroz", da Universidade de São Paulo, para obtenção do título de Mestre em Agronomia. Piracicaba, Brasil. p. 1-63.
24. VERDADE, L.M.; MOLINA, F.B. 1993. Studbook Regional do jacaré-de-papoamarelo (Caiman latirostris) 1991/1992. ESALQ/USP, Piracicaba, Brasil.

25. VERDADE, L.M.; PERINA, S.K.; MOLINA, F.B.M. 1993. Studbook Regional do jacaré-de-papo-amarelo (Caiman latirostris) 1992/1993. ESALQ/ USP, Piracicaba, Brasil.

26. VERDADE, L. M. 2000. Regression equations between body and head measurements un the broad-snouted caiman (Caiman latirostris). Rev. Brasil. Biol., 60(3): 469-482.
Endereço para Correspondência:

Mauro Sergio Cruz Souza Lima

slmauro@ufpi.edu.br

UFPI - Universidade Federal do Piauí, Campus

Amilcar Ferreira Sobral, BR 343, Km 3,5

Bairro Meladão Floriano - PI - Brasil.

CEP: 64800-000 